SuCCESSFul ENDOVASCULAR INFRARENAL ANEURYSM REPAIR IN A PATIENT With

\title{
SITUS INVERSUS TOTALIS
}

Chan YC, Ting AC, Qing KX, Cheng SW.

Division of V ascular \& Endovascular Surgery, Department of Surgery, University of Hong Kong

Medical Centre, South Wing, $14^{\text {th }}$ Floor K Block, Queen Mary Hospital, Hong Kong.

\section{AUTHOR CORRESPONDENCE:}

Dr. Yiu-Che CHAN MB BS BSc MD FRCS FRCS(General Surgery),

Division of Vascular \& Endovascular Surgery,

Department of Surgery,

University of Hong Kong Medical Centre,

South Wing, $14^{\text {th }}$ Floor K Block,

Queen Mary Hospital,

Pokfulam Road,

Hong Kong.

Tel: $\quad+852-2855-4962$

FAX: $\quad+852-2855-4961$

Email: ycchan88@hkucc.hku.hk 


\section{AbStract}

Situs inversus totalis is a rare autosomal recessive developmental anomaly. There are very few reports in the published literature of abdominal aortic aneurysm in patient with situs inversus totalis, all of whom underwent open aneurysm repair. This is the first case in the world's literature to describe a patient with situs inversus totalis who had a successful endovascular infrarenal aneurysm repair. Although endovascular infrarenal aneurysm repair should not be more challenging, the endovascular approach may decrease risk of potential errors due to unfamiliar anatomy. Technical considerations in performing endovascular procedures in patients with situs inversus totalis are discussed.

KEY WORDS: Endovascular aneurysm repair, infrarenal abdominal aortic aneurysm, situs inversus totalis, situs viscerum inversus totalis. 


\section{INTRODUCTION}

Situs inversus totalis is a rare developmental anomaly characterized by complete inversion of the thoracic and abdominal viscera, with a worldwide reported prevalence of between $0.002 \%$ and $0.01 \%$. This is not a mere clinical curiosity as recognition of concomitant anomalies (cardiac, venous, gastrointestinal, and urinary systems) is extremely important as situs inversus totalis may influence the entire surgical management approach ${ }^{2}$.

There are very few patients reported of abdominal aortic aneurysm in associated with situs inversus totalis. Kato et al. described a case of successful elective open repair of an abdominal aortic aneurysm with a false aneurysm of the right external iliac artery ${ }^{2}$. Occbionorelli et al. reported an urgent open infrarenal tube graft for a tender aneurysm in a patient with situs viscerum inversus totalis ${ }^{3}$. Similar case was reported by Baccellieri et al. using an open aorto-iliac bifurcated graft ${ }^{4}$. Kimura et al. described a successful emergency open repair of a ruptured abdominal aortic aneurysm in an eighty years old lady, requiring clamping of the descending thoracic aorta to achieve proximal aortic control ${ }^{5}$.

This is to our knowledge the first case in the world's literature on a successful elective endovascular infrarenal aneurysm repair in a patient with situs inversus totalis.

\section{CASE}

An eighty-one years old man with known situs inversus totalis and past medical history of hypertension, ischaemic heart disease and open colectomy for carcinoma of colon six years ago presented with a non-tender pulsatile expansile abdominal mass. An abdominal aneurysm was diagnosed on clinical examination, and subsequent CT scans showed a $7.5 \mathrm{~cm}$ infrarenal aortic aneurysm (FIGURE 1). After appropriate discussion with patient and with pre-operative assessment, he underwent an elective endovascular infrarenal aneurysm repair using Cook Zenith endovascular device (TFFB 26-96, right ipsilateral limb TFLE16-54, left contralateral limb TFLE20-71). The femoral arteries were accessed with open dissection. Despite tortuous and atherosclerotic external iliac arteries bilaterally, the procedure was uneventful. The aneurysm was successfully excluded and there were no endoleaks on completion angiogram. Post-operative course was unremarkable, and the patient was discharge on post-operative day three. Patient remained well and follow up CT scan (at 6 months) showed successful endovascular aneurysm repair (FIGURE 2). 


\section{DisCussion}

Situs inversus totalis is a developmental anomaly where all the major thoracic and abdominal structures are affected, with complete transposition through the sagittal plane. Although it is an autosomal recessive developmental anomaly ${ }^{6}$, patients with situs inversus totalis are phenotypically normal and lead normal healthy lives, and are usually not aware of their condition until they seek medical attention for unrelated symptoms. The reversal of the organs may lead to confusion in diagnosis, as the symptoms and signs are on the 'opposite' side. Five to ten percent of patients with situs inversus totalis have congenital heart disease, most commonly transposition of the great vessels ${ }^{7}$.

There are less than ten reported patients in world's literature on situs inversus totalis with aortic aneurysms, and this is the first reported patient who had successful endovascular aneurysm repair. There are isolated reports in the literature to suggest that abdominal aortic aneurysm occurring in the setting of congenital intestinal malrotation in an adult may be more symptomatic, manifested by intestinal obstruction ${ }^{8}$. However, this may not be the case in patients with situs viscerum inversus totalis (only the visceral organs are inverted, but the vessels are not) or situs inversus totalis, since the abdominal viscera are in the correct position, being an exact mirror image to the normal. Situs inversus totalis may influence the open aneurysm repair because of unfamiliar anatomy. These inconveniences can be bypassed using endovascular techniques.

The endovascular infrarenal aneurysm repair in patients with situs inversus totalis is essentially the same and technically should not be different from 'normal' patients. The endovascular surgeons have to re-orientate themselves in the thoracic aorta, as the descending aorta curves upwards towards the right side of the spine, and the aortic arch would appear back to front on the radiological screen. An experienced operator probably does not need to flip the radiological images on screen and can proceed as per normal, it does not really matter on what side of the spine the aorta is located.

This extremely rare case demonstrates that endovascular aneurysm repair is not only feasible but preferable in patients with situs inversus totalis with suitable anatomy. The position of the abdominal viscera (especially of the duodenum, the intestinal, the inferior vena cava, and the liver) does not influence the access to the aorta. Together with flipping of the radiological screen on the image intensifier from 'right to left', the entire endovascular procedure can be performed without technical inconvenience or difficulty. 


\section{REFERENCES:}

1. Blegen HM. Surgery in situs inversus. Ann Surg 1949; 129: 244-259.

2. Kato T, Takagi H, Sekino S, Manabe H, Matsuno Y, Umemoto T. Abdominal aortic aneurysm in situs inversus totalis. Ann Vasc Surg. 2006; 20: 267-270.

3. Occhionorelli S, Navarra G, Santini M, Carcoforo P, Sartori A, Pollinzi V, Mascoli F.Symptomatic abdominal aortic aneurysm and "situs viscerum inversus." Diagnostic and therapeutic approach. J Cardiovasc Surg (Torino). 1998; 39: 31-33.

4. Baccellieri D, Mirenda F, Mandolfino T, La Spada M, Stilo F, Spinelli F. Symptomatic aorto-iliac aneurysm and situs viscerum inversus: case report. Chir Ital. 2006; 58: 113-116.

5. Kimura N, Kawahito K, Ito S. Rupture of an abdominal aortic aneurysm in a patient with a situs inversus totalis: report of a case. Surg Today. 2008; 38: 249-252.

6. Balci S, Bostanoğlu S, Altinok G, Ozaltin F. New syndrome?: Three sibs diagnosed prenatally with situs inversus totalis, renal and pancreatic dysplasia, and cysts. $A m J$ Med Genet. 2000; 90: 185-187.

7. Perloff JK, Lindgren KM. Adult survival in congenital heart disease. 2. Uncommon defects with expected adult survival. Geriatrics. 1974; 29: 99-102.

8. Bhama JK, Ogren J, Guinn G, Fisher WE. Unique cause of duodenal obstruction by an abdominal aortic aneurysm. J V asc Surg. 2001; 34: 1130-1132. 


\section{LEGENDS FOR FIGURES}

\section{FIGURE 1}

Pre-operative three dimensional CT reconstruction ( $\mathbf{A}$ anterior and $\mathbf{B}$ right anterior-lateral views) showing $7.5 \mathrm{~cm}$ infrarenal aortic aneurysm with sinus inversus totalis. The infrarenal aneurysm neck was favourable for endovascular repair but the iliac arteries were tortuous and atherosclerotic.

\section{FIGURE 2}

Post operative three dimensional CT reconstruction (A anterior and $\mathbf{B}$ left antero-lateral views) showing successful endovascular repair. There were no endoleaks and the patient had uneventful recovery. 